\title{
Evaluation of a microbiological screening and acceptance procedure for cryopreserved skin allografts based on 14 day cultures
}

\author{
Jean-Paul Pirnay • Gunther Verween · Bruno Pascual • Gilbert Verbeken • \\ Peter De Corte $\cdot$ Thomas Rose $\cdot$ Serge Jennes - Alain Vanderkelen • \\ Miriam Marichal $\cdot$ Walter Heuninckx $\cdot$ Daniel De Vos
}

Received: 31 January 2011/ Accepted: 9 April 2011/Published online: 21 April 2011

(C) The Author(s) 2011. This article is published with open access at Springerlink.com

\begin{abstract}
Viable donor skin is still considered the gold standard for the temporary covering of burns. Since 1985, the Brussels military skin bank supplies cryopreserved viable cadaveric skin for therapeutic use. Unfortunately, viable skin can not be sterilised, which increases the risk of disease transmission. On the other hand, every effort should be made to ensure that the largest possible part of the donated skin is processed into high-performance grafts. Cryopreserved skin allografts that fail bacterial or fungal screening are reworked into 'sterile' non-viable glycerolised skin allografts. The transposition of the European Human Cell and Tissue Directives into Belgian Law has prompted us to install a pragmatic microbiological screening and acceptance procedure, which is based on 14 day enrichment broth cultures of finished product samples and treats the complex issues of 'acceptable bioburden' and 'absence
\end{abstract}

J.-P. Pirnay $(\bowtie) \cdot$ G. Verween · B. Pascual ·

G. Verbeken - P. De Corte - T. Rose .

S. Jennes - A. Vanderkelen - D. De Vos

Human Cell and Tissue Banks, Laboratory for Molecular

and Cellular Technology, Burn Wound Centre, Queen

Astrid Military Hospital, Brussels, Belgium

e-mail: jean-paul.pirnay@mil.be

M. Marichal

Department of Pathology, Vrije Universiteit Brussel,

Brussels, Belgium

W. Heuninckx

Clinical Laboratory, Queen Astrid Military Hospital,

Brussels, Belgium of objectionable organisms'. In this paper we evaluate this procedure applied on 148 skin donations. An incubation time of 14 days allowed for the detection of an additional $16.9 \%(25 / 148)$ of contaminated skin compared to our classic 3 day incubation protocol and consequently increased the share of non-viable glycerolised skin with $8.4 \%$. Importantly, $24 \%$ of these slow-growing microorganisms were considered to be potentially pathogenic. In addition, we raise the issue of 'representative sampling' of heterogeneously contaminated skin. In summary, we feel that our present microbiological testing and acceptance procedure assures adequate patient safety and skin availability. The question remains, however, whether the supposed increased safety of our skin grafts outweighs the reduced overall clinical performance and the increase in work load and costs.

Keywords Allograft donor skin - Cell and tissue banking - Cryopreservation - Bacteriological and fungal contamination $\cdot$ Screening

\section{Introduction}

Human allograft skin is most often obtained from cadaveric donors and used to bring about a temporary-it will inevitably be rejected-wound closure on full thickness burn or chronic wounds. It acts as a 
mechanical and biological barrier, decreasing the loss of water, protein, electrolytes and heat through the wound and decreasing the risk of infection. It is also known to decrease wound pain and the frequency of dressing changes (Britton-Byrd et al. 2008).

Fresh viable cadaveric skin is considered the best biologic dressing for burns because it provides a faster and more durable adherence to the wound bed and a better control of microbiological contamination than preserved skin (Greenleaf et al. 1991; Cinamon et al. 1993; Pianigiani et al. 2005). A major concern with the use of fresh cadaveric skin is the risk of disease transmission (Kearney 2005; Pianigiani et al. 2006). To enable a better availability and a more extensive quality and safety control (ample time for screening), skin allografts are often preserved in skin banks. Human allograft skin can be preserved by numerous methods: cool storage $\left(2-8^{\circ} \mathrm{C}\right)$, cryopreservation $\left(-20\right.$ to $\left.-196^{\circ} \mathrm{C}\right)$, deep freezing, freeze drying or dehydration using high-concentration solutes like glycerol (Kearney 2005). These methods amount to different ranges of allograft skin viability, integrity, microbiological contamination and immunogenicity (Ingham et al. 1993; Hettich et al. 1994; Richters et al. 1997; van Baare et al. 1998; Bravo et al. 2000; Saegeman et al. 2008). Glycerol preserved allograft skin, for example, is non-viable and has been used successfully in burn surgery in the past (Kreis et al. 1989; de Backere 1994; Huang et al. 2004). Cryopreservation is considered to be the best method for the long-term preservation of skin (Kearney 2005). Cryopreserved cadaveric skin exhibits a certain level of viability and engrafts to the wound bed, forming an excellent substrate for revascularization and recolonisation by host cells (Aggarwal et al. 1985; Cinamon et al. 1993, Kearney 2005). Unfortunately, cryopreservation also supports bacterial and fungal survival, which can be problematic in the case of skin allograft preservation. Pianigiani et al. recently (2010) discovered that microbiological contamination of donor skin is significantly affected by the type of processing (cryo- or glycerol preservation), with highest levels of contamination found in cryopreserved donor skin. In contrast to most harvested donor tissues (e.g. musculoskeletal tissue and heart valves), skin tissue is inherently colonised by a substantial amount of commensal microorganisms and thus non-sterile at the time of harvesting. As superficial decontamination of the donor sites before harvesting, using antiseptics, is not indefectible, freshly harvested donor skin is often collected in an antibiotics containing transport medium (Kearney 2005).

Unfortunately, sterilisation techniques cannot be applied for viable cryopreserved skin as they inactivate the skin cells (Kearney 2005).

The microbiological evaluation of inherently nonsterile human cryopreserved skin is much more complex than for sterile products. It is therefore very difficult to promulgate functionally realistic and ethically acceptable guidelines for the evaluation of cryopreserved donor skin for bacteria and fungi and for their acceptability for clinical use.

Ideally, the microbiological screening strategy for cryopreserved skin allografts should ensure a total absence of 'relevant pathogens' as well as the absence of substantial bioburdens of inherent skin commensals.

Historically, in Belgium as in many other countries, the microbiological contamination of human skin allografts was mostly assessed using routine clinical microbiology methods, and more specific 2-3 day skin cultures. In December 2008, the European Human Cell and Tissue Directives (2004/23/CE, 2006/17/CE and 2006/86/CE) were transposed to Belgian Law. To be accredited, Belgian human celland tissue banks have to comply with quality and safety criteria defined in Royal Decrees (September 28, 2009). In addition, the Belgian Superior Health Council published (October 1, 2008) practical and detailed quality and safety criteria for human allografts. According to these criteria, presence of pathogens (Table 1) in finished product samples results in a definite rejection of the donor tissue if no validated sterilisation or decontamination method is applied. Skin allografts can however be accepted for clinical use, without decontamination, when the bacteriological and mycological cultures only reveal low bioburdens of inherent inhabitants of the residential skin flora. While this bioburden can be determined using elaborate and expensive quantitative cultures, it is permitted to use a more pragmatic approach based on adequate 14 day microbiological cultures. If these cultures are negative and all other quality and safety criteria are met, skin allografts can be released for clinical use. If microbiological 
growth is detected (increased turbidity) during the first 7 days of incubation, a relatively high bioburden is suspected and the tissue must be rejected, sterilized or decontaminated using a validated method. If growth is detected after this period (i.e. days 8-14), the growing microorganism(s) need(s) to be identified. Identification of micro-organisms considered to be pathogenic (Table 1) again implies rejection, sterilisation or decontamination. If a common (e.g. exhibiting no particular antibiotic resistance or virulence) member of the skin flora is identified as sole contaminant, and all other quality requirements are met, the cryopreserved skin allografts can be released as such.

If a cocktail of antibiotics was used during the transport and/or conditioning process, those drugs need to be neutralised or washed away before the actual bacteriological and mycological screening procedure is started.

Skin donations that do not meet the quality and safety criteria have to be destroyed (traceable) unless an explicit informed consent for research use was obtained from the donor, his legal representative or a medical ethical committee.

The Brussels military skin bank preferably processes cadaveric skin into viable cryopreserved allografts. Cryopreserved skin that does not meet

Table 1 Non-limiting list of microorganisms, which should not be present in the skin allograft

Bacteria
Acinetobacter baumannii
Beta-hemolytic streptococci
Burkholderia cepacia
Clostridium perfringens
Clostridium tetani
Corynebacterium diphteriae
Enterobacteriaceae (coliforms)
Pseudomonas aeruginosa
Staphylococcus aureus
Fungi
Aspergilus spp.
Candida spp.
Mucor spp.
Penicillium spp.
Other yeasts and fungi
Mycobacteria (at risk donors)

the microbiological acceptance criteria is reworked by glycerol-decontamination (Verbeken et al. 2011). As such, the bank provides cryopreserved as well as glycerolised skin, whilst assuring an optimal use of the scarce donor skin. In the summer of 2008 we introduced a microbiological screening and acceptance procedure for cryopreserved skin allografts based on 14 day cultures of finished product samples. Since then 148 cadaveric skin donations were processed. With the exception of the transport medium, which was updated in the course of 2009, all donations were processed in an identical way.

In this paper we present the results of a retrospective evaluation of our specific microbiological screening and acceptance procedure.

\section{Materials and methods}

\section{Skin procurement}

Skin from 148 cadaveric donors was obtained under aseptic conditions in an autopsy room or operation theatre. Skin allografts and blood samples (serological screening) were recovered within respectively 48 and $24 \mathrm{~h}$ after death. The donors were refrigerated $\left(2-8^{\circ} \mathrm{C}\right)$ within $6 \mathrm{~h}$ after death. The donor sites, most often the back and the legs, were shaven and thoroughly scrubbed with $7.5 \%$ polyvidon-iodine soap (Iso-Betadine Savon Germicide, Meda Pharma, Belgium), rinsed with tap water and disinfected with $0.5 \%(\mathrm{w} / \mathrm{v})$ chlorhexidine and $70 \%(\mathrm{v} / \mathrm{v})$ isopropanol solution (Hibitane Plus, Mölnlycke Health Care, UK). Non donor sites were covered with sterile operative fields. The donor sites were lubricated with sterile liquid paraffin (Fagron, Belgium) and skin strips (approximately $300 \times 50 \times 0.3 \mathrm{~mm}$ ) were removed using a battery operated dermatome (Aesculap, Germany). The donor skin was collected in a sterile recipient (Maco Biotech storage pot, MacoPharma, Belgium) containing sterile transport medium and transported, on crushed ice, to the skin bank. Two transport media (TM) were used. The first 57 skin donations were transported in TM1 (PAA Laboratories $\mathrm{GmbH}$, Austria), which consisted of Penicillin (100,000 units/1), Streptomycin (100,000 units/1) and Amphotericin B $(1.25 \mathrm{mg} / \mathrm{l})$ in Medium for the Culturing of Epithelial Cells (MCEC). The following 91 skin donations were transported in TM2, which 
was composed of $800 \mathrm{ml}$ MCEC (Invitrogen Life Technology, Belgium) supplemented with $200 \mathrm{ml}$ of Cambridge Antibiotic Solution (CAS, Inverclyde Biologicals Ltd, UK). CAS consisted of Gentamicin sulphate (4 g/l), Imipenem/cilastin Na (0.2 g/l), Polymyxin B sulphate $(0.2 \mathrm{~g} / \mathrm{l})$, Vancomycin $\mathrm{HCl}(0.05 \mathrm{~g} /$ 1) and Nystatin $(2,500,000$ units/l) in Medium 199 with $25 \mathrm{~mm}$ HEPES.

\section{Skin processing and sampling}

Upon arrival in the skin bank, skin allografts were kept in TM for min. 24 and max. $72 \mathrm{~h}$ at $2-8^{\circ} \mathrm{C}$ prior to further processing. Processing was performed according to processing instructions and conform with national regulations and guidelines, in a GMP grade A laminar air flow cabinet in a GMP grade $\mathrm{C}$ environment. To wash away the antibiotics, skin strips were incubated for $1 \mathrm{~h}$ at room temperature in at least $0.2 \mathrm{ml} / \mathrm{cm}^{2}$ of sterile $0.9 \%$ sodium chloride solution (Vioser S. A., Greece).

After the washing step, a representative skin sample consisting of 20 randomly selected skin pieces of approximately $2 \mathrm{~cm}^{2}$ (1-2\% of the skin donation), were obtained aseptically and transferred to a recipient with $25 \mathrm{ml}$ of thioglycollate broth with resazurin (bioMérieux, Belgium) and to a recipient with $25 \mathrm{ml}$ of Sabouraud broth (bioMérieux, Belgium) (10 skin pieces per recipient) for in-process bacteriological and mycological testing.

Skin strips were then trimmed and impregnated, for $30 \mathrm{~min}$, in sterile cryopreservation medium consisting of $30 \%(\mathrm{v} / \mathrm{v})$ glycerol (Pharma Belgium) in Hartmann solution (Baxter, Belgium) and transferred to sterile aluminum foil (Aluminum foil 8011A, Alcomet AD, Bulgaria). Skin pieces were spread (dermis side up) onto the foil and measured. An inventory of all skin strips was made and recorded. Each aluminum foil support held approximately $300 \mathrm{~cm}^{2}$ of donor skin. After addition of an extra $5 \mathrm{ml}$ of cryopreservation medium, each aluminum foil support was folded and seal-packed in a sterile laminated aluminum inner (CE class IIa Maco BioTech Freezing Bag, MacoPharma, Belgium) and outer (ATMI LifeSciences, Belgium) pouch. An extra package containing a representative skin sample (see above) for microbiological testing of the finished product was made. The skin pouches were frozen using a computerized biological freezer (Kryo Planer

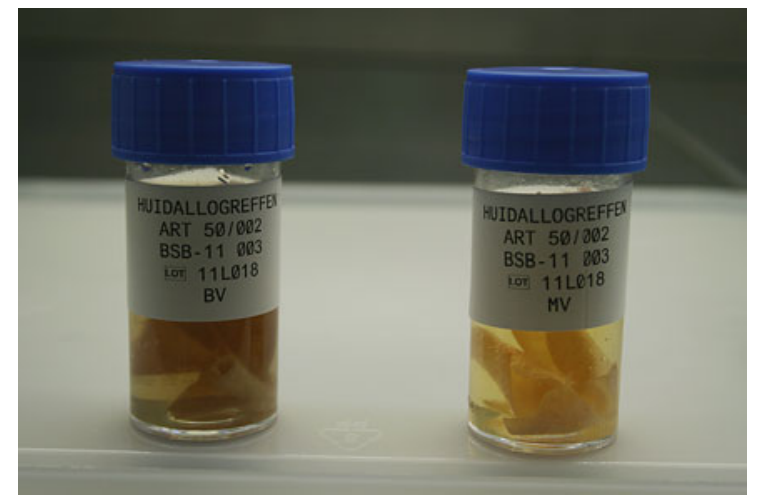

Fig. 1 Skin samples for bacteriological and mycological testing

MRV controller, Cryo Solutions, The Netherlands). Cooling rates were $-1{ }^{\circ} \mathrm{C} / \mathrm{min}$ towards $-6^{\circ} \mathrm{C}$, followed by $-5^{\circ} \mathrm{C} / \mathrm{min}$ towards $-30^{\circ} \mathrm{C}$ and $-20^{\circ} \mathrm{C} / \mathrm{min}$ towards $-140^{\circ} \mathrm{C}$. Skin pouches were then transferred to the vapour phase of liquid nitrogen $\left(<-135^{\circ} \mathrm{C}\right)$ for long term storage. The microbiological test pouch was thawed in a $37^{\circ} \mathrm{C}$ water bath and the skin pieces were rinsed in at least $1 \mathrm{ml} / \mathrm{cm}^{2}$ of sterile $0.9 \%$ sodium chloride solution (Vioser S. A., Greece) prior to transfer to recipients with appropriate media (see above) for bacteriological and mycological testing (Fig. 1). 'In-process' and 'finished product' skin samples were immediately sent to the clinical laboratory of the Queen Astrid Military Hospital, which is accredited for microbiological testing.

\section{Microbiological cultures}

In the clinical laboratory, skin samples were immediately analysed for bacteriological and fungal contamination using an extensive clinical microbiology methodology (Fig. 2), designed to recover aerobic and anaerobic as well as fast- and slow-growing microorganisms. In short, the skin samples were cultured for 14 days at appropriate temperatures and examined daily for visual evidence of growth (turbidity). Skin samples were not disrupted (e.g. by stomaching or sonicating) as this caused the medium to appear turbid, making the detection of microorganism growth difficult. We assumed that 14 days was enough time for the bacteria to migrate from the adnexal structures to the edges of the skin samples. 


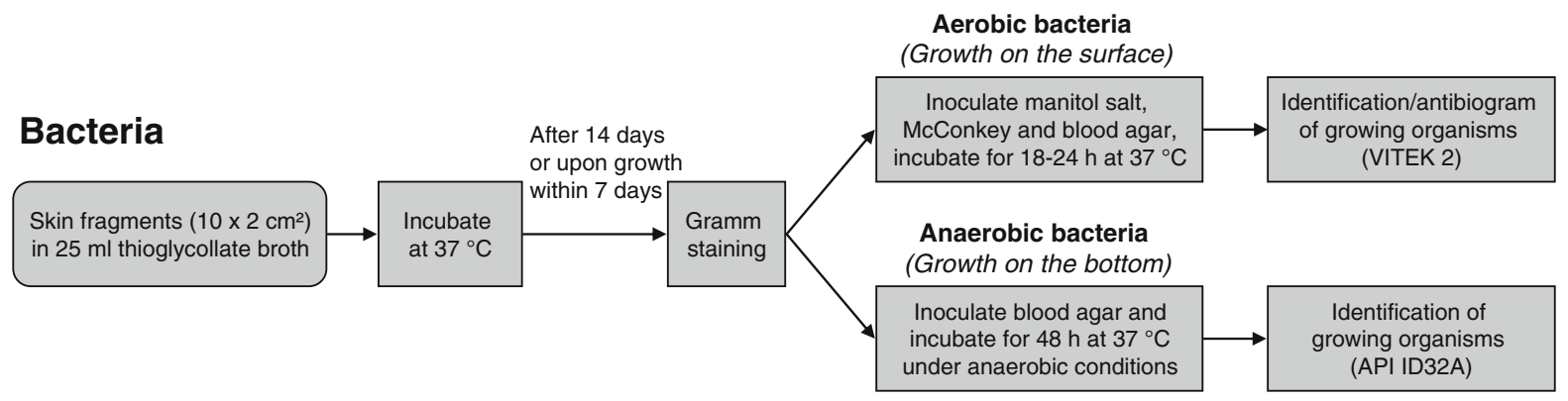

\section{Fungi and yeasts}

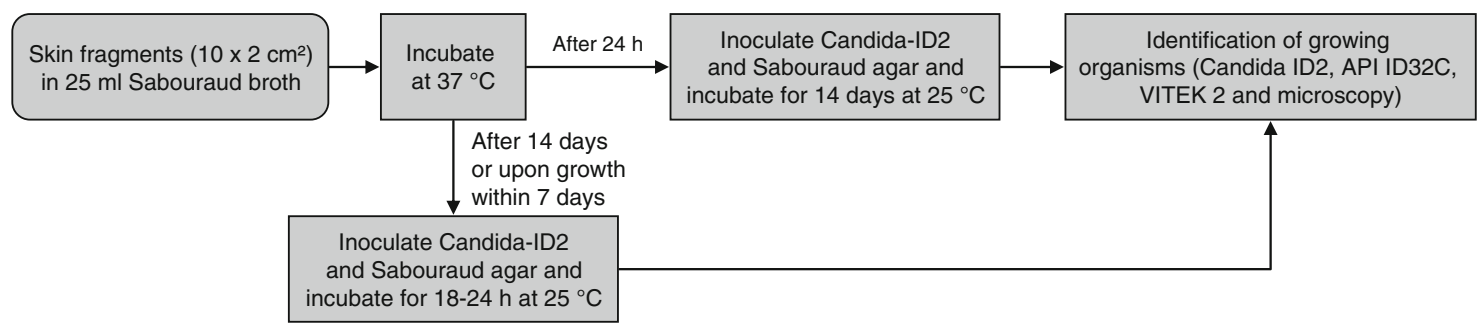

Fig. 2 Overview of the clinical microbiology methodology for the testing of skin samples for bacteria, fungi and yeasts

After these 14 days and whenever growth was observed, different agar plates (Manitol Salt, McConkey, Blood, Candida-ID2 and Sabouraud agar, bioMérieux, Belgium) were inoculated and incubated at appropriate temperatures (see Fig. 2). Plates for anaerobes were incubated under anaerobic conditions (Anaerogen compact, Oxoid, Belgium). Cultures showing growth were identified to the genus and species level using selective media (Candida-ID2 agar), automated biochemical tests (VITEK 2, API ID32A or C, all from bioMérieux, Belgium) or, in the case of filamentous fungal colonies, microscopic observation. Antibiotic susceptibility was also assessed with the VITEK 2 system.

\section{Acceptance procedure}

The acceptance or eventual glycerol-decontamination or destruction of cryopreserved skin allografts depends on the magnitude of bacterial load and the presence or absence of pathogenic microorganisms, factors which are derived from 14 day cultures of finished product samples. This complex procedure is graphically represented in a decision tree (Fig. 3).

\section{Results and discussion}

In light of the chronic shortage of human skin for burn wound treatment and out of respect for the donor and his family, every effort should be made to ensure that donated human skin does not go to waste. In view of an optimal conservation of clinically relevant properties of skin allografts, we strive to provide the physicians with as much high quality cryopreserved donor skin as possible. These choices entail the complex issues of 'acceptable bioburden' and 'absence of objectionable organisms'.

In 2008 we introduced a pragmatic microbiological screening and acceptance procedure for cryopreserved skin allografts based on 14 day cultures of the finished product samples, i.e. pieces of skin allografts that have undergone all stages of production, including packaging, cryopreservation and labelling. This procedure was analogous to common drug manufacturing practices and compliant with the relevant national regulations, standards and guidelines. In this paper we evaluate this procedure applied on 148 skin donations. As could be expected, antibiotics were not successful in a significant part of the skin retrievals. At $2-8^{\circ} \mathrm{C}$, TM1 failed to decontaminate no less than $47.4 \%(27 / 57)$ of skin donations and even the broad 


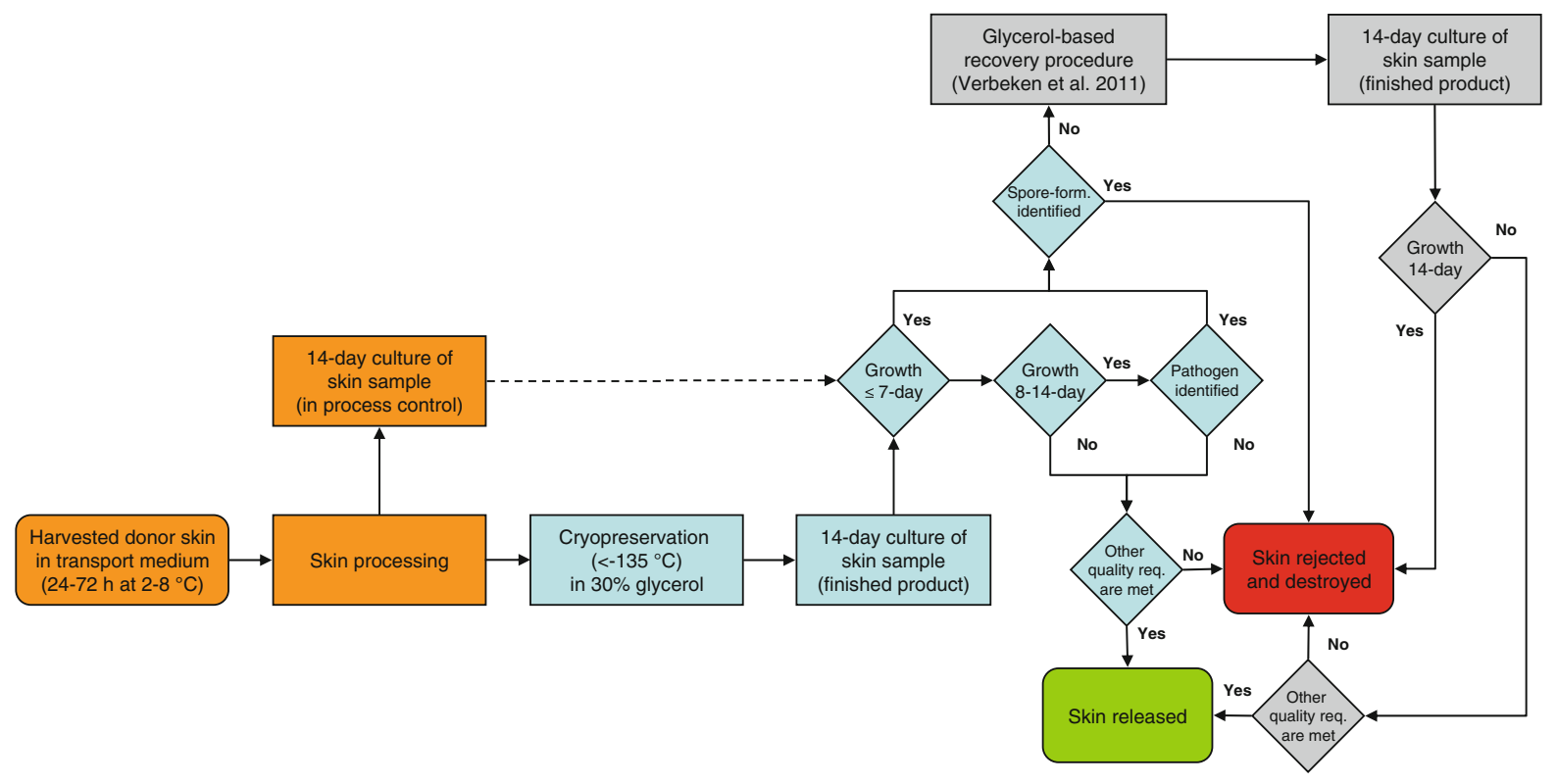

Fig. 3 Decision tree of the acceptance procedure for skin allografts

Table 2 Number of positive cultures in relation to transport medium and culture period

\begin{tabular}{|c|c|c|c|}
\hline \multirow[t]{2}{*}{$\begin{array}{l}\text { Culture period } \\
\text { (days) }\end{array}$} & \multicolumn{3}{|c|}{$\begin{array}{l}\text { Number of donations with positive culture } \\
(\%)\end{array}$} \\
\hline & $\begin{array}{l}\text { TM1 } \\
(\mathrm{n}=57)\end{array}$ & $\begin{array}{l}\text { TM2 } \\
(\mathrm{n}=91)\end{array}$ & $\begin{array}{l}\text { Total } \\
(\mathrm{n}=148)\end{array}$ \\
\hline$\leq 2$ & $13(22.8)$ & $6(6.6)$ & $19(12.8)$ \\
\hline$>2 \leq 7$ & $5(8.8)$ & $4(4.4)$ & $9(6.1)$ \\
\hline$>7 \leq 14$ & $9(15.8)$ & $7(7.7)$ & $16(10.8)$ \\
\hline Total & $27(47.4)$ & 17 (18.7) & $44(29.7)$ \\
\hline
\end{tabular}

spectrum antibiotic containing TM2 solution was not capable of decontaminating 17/91 (18.7\%) skin batches (Table 2). Antibiograms revealed that these bacteria were sensitive (in vitro) to at least one of the antibiotics present in the CAS-cocktail. Rooney et al. (2008) reported that approximately $22 \%$ of skin allografts were not reliably decontaminated by antibiotic treatment. There are two likely causes as to why antibiotic cocktails are not efficient in some cases. First of all, bacteria can be hidden deep into the harvested skin (e.g. in the dept of the hair-follicles) where the antibiotics can't reach them in due time and, secondly, the optimal operating temperature of numerous antibiotics is much closer to $37^{\circ} \mathrm{C}$ than to $2-8^{\circ} \mathrm{C}$ (Kearney 2005). We are therefore currently evaluating the inclusion of an additional short (to limit cell inactivation) antibiotic incubation step at $37^{\circ} \mathrm{C}$.

Classical 3 day cultures allowed for the detection of contamination in $12.8 \%$ (19/148) of skin retrievals (Table 2; Fig. 4). Extension of the culture period to 14 days revealed contamination in an additional 25 (16.9\%) skin donations, bringing the total to 44 (29.7\%) (Table 2; Fig. 4).

As reported by other authors (Ireland and Spelman 2005; Neely et al. 2008; Pianigiani et al. 2010), the most commonly isolated microorganisms were common skin contaminants like coagulase negative staphylococci, especially Staphylococcus epidermidis (35.4\% of positive cultures), but occasionally coliforms like Eschericchia coli $(6.3 \%)$ and Klebsiella pneumoniae $(6.3 \%)$ and recognised bacterial and fungal pathogens like Candida albicans (10.4\%), Pseudomonas aeruginosa (4.1\%) and Staphylococcus aureus $(4.1 \%)$ were isolated (Table 3). Forty-three percent (19/44) of contaminations were revealed after $48 \mathrm{~h}$ cultures, while the remaining $56.8 \%$ (25/44) of contaminations were only revealed after at least 3 days of incubation (Fig. 4). Britton-Byrd et al. (2008) analysed 7 day cultures in 735 skin donations and reported that only one had positive cultures post 3 days. They consequently concluded that 3 day microbiologic cultures are as safe as 7 day cultures. 


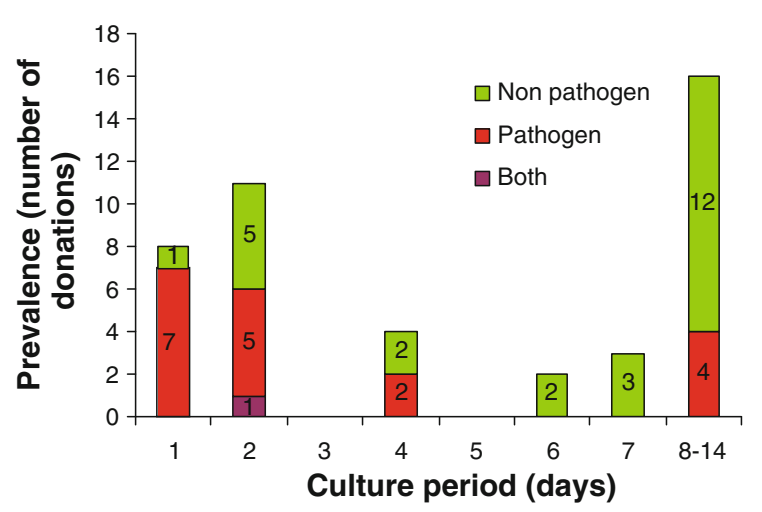

Fig. 4 Skin culture results in relation to the culture period

Table 3 Skin contaminants and their prevalence

\begin{tabular}{lc}
\hline Micro-organism & $\begin{array}{l}\text { Prevalence (Number } \\
\text { of donations) }\end{array}$ \\
\hline Pathogens (see Table 1) & 20 \\
Candida albicans & 5 \\
Escherichia coli & 3 \\
Klebsiella pneumoniae & 3 \\
Staphylococcus aureus & 2 \\
Pseudomonas aeruginosa & 2 \\
Enterococcus faecalis & 2 \\
Clostridium perfringens & 1 \\
Enterobacter cloacae & 1 \\
Enterococcus faecium & 1 \\
Non pathogens & 28 \\
Staphylococcus epidermidis & 17 \\
Staphylococcus capitis & 5 \\
Staphylococcus warneri & 2 \\
Corynebacterium minutissimum & 1 \\
Propionibacterium acnes & 1 \\
Staphylococcus coagulase negative & 1 \\
Staphylococcus simulans & 1 \\
\hline
\end{tabular}

Interestingly, we observed that $16(64 \%)$ of these post 3 day culture positives only appeared post 8 days of incubation. Most of the slow growers were skin commensals, like propionibacteria, which are known to be slow-growers. Not negligible, however, is the fact that $24 \%(6 / 25)$ of these slow-growers were considered to be potentially pathogenic (C. albicans, $P$. aeruginosa. S. aureus, E. faecium and K. pneumoniae) (Fig. 4). In accordance with our acceptance procedure (Fig. 3), these contaminated skin batches were glycerol-decontaminated and it was thus not possible to investigate the clinical relevance of these post 3 day positive cultures.

In $27.3 \%(12 / 44)$ of culture positive skin procurements an 8-14 day culture period revealed the presence of only commensals (Fig. 3). Ten of those were released in the form of cryopreserved allografts, two were destroyed, one due to a positive serology, another due to a belatedly discovered donor malignancy.

The remaining 32 culture positive skin batches were reworked using a validated glycerol-based decontamination protocol (Verbeken et al. 2011) and were again subjected to microbiological screening (Fig. 3). Twenty-nine were released in the form of non-viable glycerolised skin, two were destroyed after emergence of the endospore-forming bacterium Bacillus subtilis during the decontamination procedure, one was destroyed due to a non-conform blood sample. It is plausible that B. subtilis remained dormant or was overgrown by the other contaminating flora in the initial microbiological screening, but was respectively reactivated or selected by the glycerolisation process. Note that using 3 day cultures, as was the case until mid 2008, would have given rise to the glycerol-decontamination of 19 skin donations.

An additional issue that came up during our evaluation was the one of 'relevant sampling'. In pharmaceutical product manufacturing it is assumed that the distribution of microbiological contamination is homogeneous throughout the product. This allows for a 'representative' sample for microbiological evaluation that represents a small part of the batch. However, in practice, it is well-known that microbiological contamination is never homogeneous and certainly not when it comes to human donor skin, which is not of uniform consistency, composition and-most important in this context-bacterial load, and can not be homogenized (e.g. by mixing). This issue surfaced when reviewing the results of the inprocess microbiological tests. The 14 day cultures of the skin sampled prior to the cryopreservation procedure revealed an extra $14(9.5 \%)$ contaminated donations, including 8 with a pathogen listed in Table 1. It is likely that at least a part of these contaminating microorganisms survived cryopreservation and consequently did not come to light during the microbiological screening of the finished product. 
We suspect that even a sample consisting of $1-2 \%$ of the batch does not allow for a watertight microbiological screening of very unevenly contaminated skin and consider revising our sampling procedure. The clinical relevance of these contaminations, which potentially eluded the microbiological screening procedure, was investigated. No adverse reactions were reported and a review of the medical files of the 18 acceptor patients showed no indications of a possible transfer of microorganisms from the skin grafts. For three patients the infecting species matched those identified in the 'in process' samples, but the infection and graft sites and dates did not match. These findings correspond with the report of Neely et al. (2008). They analysed the cultures of 61 skin donations and 38 acceptor patients and found that none of microbes isolated from any burn patient allograft site matched microorganisms from donor allograft cultures.

Even so, we are considering the integration of these in-process microbiological cultures in the acceptance procedure of cryopreserved skin grafts (dotted line in Fig. 3).

In summary, $70.3 \%(104 / 148)$ of skin donations showed negative 14 day cultures and could be considered to be 'sterile', in analogy with sterility tests in pharmaceuticals, which also require 14 day incubation. An additional 6.8\% (10/148) of skin donations exhibited a low commensal bioburden and could be considered to be 'aseptic' (free from pathogenic microorganisms), which is at least compatible with the microbiological qualification of the setting where most of the grafts will eventually end up, i.e. a nonsterile (burn) wound in an aseptic environment (e.g. an operating theatre). Obviously, this reasoning does not apply for human tissues that are initially sterile (e.g. musculoskeletal tissue and heart valves) and are grafted inside the body. Successful glycerol-decontamination resulted in the release of an extra $19.6 \%$ (29/148) of skin donations in the form of glycerolised skin allografts. These non-viable glycerolised skin allografts are used for some specific indications or when cryopreserved viable skin is not available. Finally, only two $(1.4 \%)$ skin donations failed the microbiological acceptance criteria and were destroyed.

Although there are no clear indications that the use of 14 day microbiologic cultures is safer than 3 day cultures, when the culture results are used as the basis of the acceptance procedure described in this paper
(Fig. 3), they did not give rise to an increase in rejection rate, but they did effectuate an increase in the percentage of donations that had to be released as less effective non-viable glycerolised skin allografts from $12.8 \%$ (19/148) to $21.6 \%$ (32/148). We feel that our microbiological testing and acceptance procedure assures patient safety and skin availability. The question remains, however, whether the potentially increased safety of our skin grafts outweighs the reduced overall clinical performance and the increase in work load and costs associated with the extended processing and microbiological testing procedure.

Acknowledgments The authors wish to thank Ms Magda Pauwels, Ms Christel Taquet, Ms Jana Kaczynski and $\mathrm{Mr}$ Pascal Swerts for excellent technical assistance.

Open Access This article is distributed under the terms of the Creative Commons Attribution Noncommercial License which permits any noncommercial use, distribution, and reproduction in any medium, provided the original author(s) and source are credited.

\section{References}

Aggarwal SJ, Baxter CR, Diller KR (1985) Cryopreservation of skin: an assessment of current clinical applicability. J Burn Care Rehabil 6:469-476

Bravo D, Rigley TH, Gibran N, Strong DM, Newman-Gage H (2000) Effect of storage and preservation methods on viability in transplantable human skin allografts. Burns 26:367-378

Britton-Byrd BW, Lynch JP, Williamson S, McCauley RL (2008) Early use of allograft skin: are 3 day microbiologic cultures safe? J Trauma 64:816-818

Cinamon U, Eldad A, Chaouat M, Wexler MR, Israeli A, Zagher U, Ben-Bassat H (1993) A simplified testing system to evaluate performance after transplantation of human skin preserved in glycerol or in liquid nitrogen. J Burn Care Rehabil 14:435-439

de Backere AC (1994) Euro skin bank: large scale skinbanking in Europe based on glycerol-preservation of donor skin. Burns 20:S4-S9

Greenleaf G, Cooper ML, Hansbrough J (1991) Microbial contamination in allografted wound beds in patients with burns. J Burn Care Rehabil 12:442-445

Hettich R, Ghofrani A, Hafemann B (1994) The immunogenicity of glycerol-preserved donor skin. Burns 20:S71S76

Huang Q, Pegg DE, Kearney JN (2004) Banking of non-viable skin allografts using high concentrations of glycerol or propylene glycol. Cell Tissue Bank 5:3-21

Ingham E, Matthews JB, Kearney JN, Gowland G (1993) The effects of variation of cryopreservation protocols on the immunogenicity of allogeneic skin grafts. Cryobiology 30:443-458 
Ireland L, Spelman D (2005) Bacterial contamination of tissue allografts-experiences from the donor tissue bank of Victoria. Cell Tissue Bank 6:181-189

Kearney JN (2005) Guidelines on processing and clinical use of skin allografts. Clin Dermatol 23:357-364

Kreis RW, Vloemans AF, Hoekstra MJ, Mackie DP, Hermans RP (1989) The use of non-viable glycerol-preserved cadaver skin combined with widely expanded autografts in the treatment of extensive third-degree burns. J Trauma 29:51-54

Neely AN, Plessinger RT, Stamper B, Kagan RJ (2008) Can contamination of a patient's allograft be traced back to the allograft donor? J Burn Care Res 29:73-76

Pianigiani E, Ierardi F, Di Simplicio FC, Andreassi A (2005) Skin bank organization. Clin Dermatol 23:353-356

Pianigiani E, Risulo M, Ierardi F, Sbano P, Andreassi L, Fimiani M, Caudai C, Valensin PE, Zazi M (2006) Prevalence of skin allograft discards as a result of serological and molecular microbiological screening in a regional skin bank in Italy. Burns 32:348-351

Pianigiani E, Ierardi F, Cuciti C, Brignali S, Oggioni M, Fimiani M (2010) Processing efficacy in relation to microbial contamination of skin allografts from 723 donors. Burns 36:347-351

Richters CD, Hoekstra MJ, van Baare J, du Pont JS, Kamperdijk EW (1997) Immunogenicity of glycerol-preserved human cadaver skin in vitro. J Burn Care Rehabil 18: 228-233

Rooney P et al (2008) Sterilisation of skin allograft with gamma irradiation. Burns 34:664-673

Saegeman VS, Ectors NL, Lismont D, Verduyckt B, Verhaegen J (2008) Short- and long-term bacterial inhibiting effect of high concentrations of glycerol used in the preservation of skin allografts. Burns 34:205-211

van Baare J, Ligtvoet EE, Middelkoop E (1998) Microbiological evaluation of glycerolized cadaveric donor skin. Transplantation 65:966-970

Verbeken G, Verween G, De Vos D, Pascual B, De Corte P, Richters C, De Coninck A, Roseeuw D, Ectors N, Rose T, Jennes S, Pirnay JP (2011) Glycerol treatment as recovery procedure for cryopreserved human skin allografts positive for bacteria and fungi. Cell Tissue Bank. [Epub ahead of print] 